\title{
Three-dimensional few-cycle optical pulses in germanene with damping and amplification
}

\author{
Natalia Konobeeva ${ }^{1, *}$, and Mikhail Belonenko ${ }^{1,2}$ \\ ${ }^{1}$ Volgograd State University, Volgograd, 400062 Russia \\ ${ }^{2}$ Volgograd Institute of Business, Volgograd, 400048 Russia
}

\begin{abstract}
We investigate three-dimensional few-cycle optical pulse propagation in germanene. We obtain an effective equation for description of the multidimensional pulses dynamics with damping and amplification. We study the effects which are observed with the change in the initial pulse shape at the entrance to the sample.
\end{abstract}

In recent years, the graphene-like structures generated increased interest from researchers because of the unique properties valuable in many practical applications. Such materials include, for example, germanene. It is a single layer structure constructed of germanium atoms, which are located at the sites of the hexagonal lattice [1]. This material was predicted by scientists in 2009, and only in 2014 it was synthesized. The main distinguishing feature of germanene from other graphene-like materials is a strong spin-orbit interaction, which is three orders of magnitude greater than the spin-orbit interaction in graphene. Therefore, it leads to a significant impact on the few-cycle pulse propagation in this medium.

Let us write the dispersion law for germenene [2]:

$$
\varepsilon_{\sigma \xi}= \pm \sqrt{v^{2} k^{2}+\frac{1}{4}\left(\Delta_{z}-\sigma \xi \Delta_{S O}\right)^{2}}
$$

where $\sigma$ is the electron spin, $v$ is the velocity of Dirac electrons, $\mathbf{k}=\left(k_{x}, k_{y}\right)$ is the quasimomentum of the electrons, $\Delta_{\text {So }}$ is the spin-orbit interaction, $\Delta_{\mathrm{z}}$ is the potential on the one site, $\Delta_{\mathrm{z}}=E_{z} d, d$ is the distance between the sublattice planes, $\sigma_{\mathrm{i}}, \tau_{\mathrm{i}}$ are the Pauli matrices [3].

Using similar arguments as in [4], we can write the equation for the pulse propagation in cylindrical coordinate system as follows:

$$
A_{t t}-\Gamma A_{t}=\frac{1}{r} \frac{\partial}{\partial r}\left(r \frac{\partial A}{\partial r}\right)+\frac{\partial^{2} A}{\partial z^{2}}+\frac{1}{r^{2}} \frac{\partial^{2} A}{\partial \phi^{2}}+4 \pi j(A) \exp \left(-t / t_{r e l}\right)
$$

here the current density $\mathrm{j}$ is calculated using the electron spectrum (1), $A$ is the vectorpotential, parameter $\Gamma$ describes the pumping of the electric field and, consequently, its amplification, $c=1$ is the light velocity. Here, the relaxation of electrons with time $t_{\text {rel }}$ is phenomenologically taken into account, which leads to a decay of the current.

Equation (2) was solved numerically using classical numerical scheme. The initial condition in the three-dimensional case was chosen as a very short pulse, consisting of one, and two oscillations ( $n=1$ and 2 respectively). The evolution of the electromagnetic field upon

Corresponding author: yana nn@,volsu.ru 
its spreading over a sample is shown in Fig. 1. The dependence of the pulse shape on the different initial velocity is presented in Fig. 2.

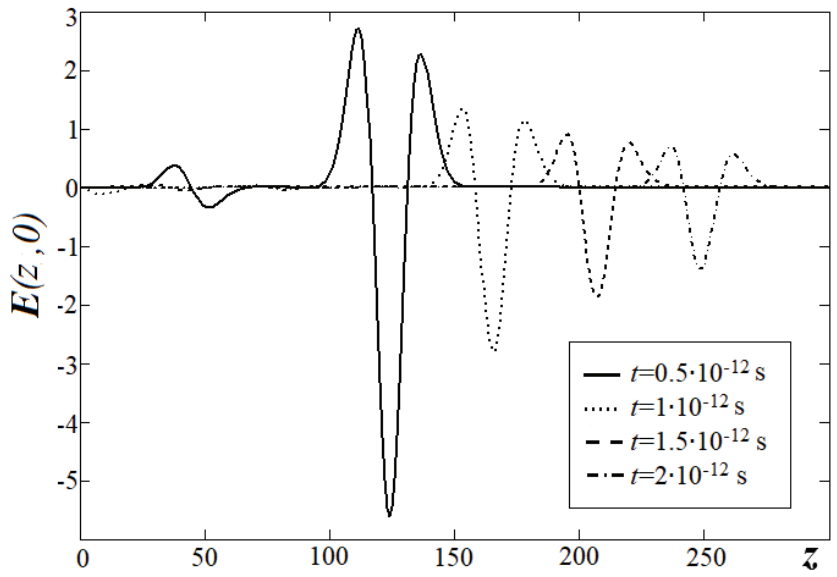

Fig. 1. The electric field at different instances of time at the $r=0, n=1$.
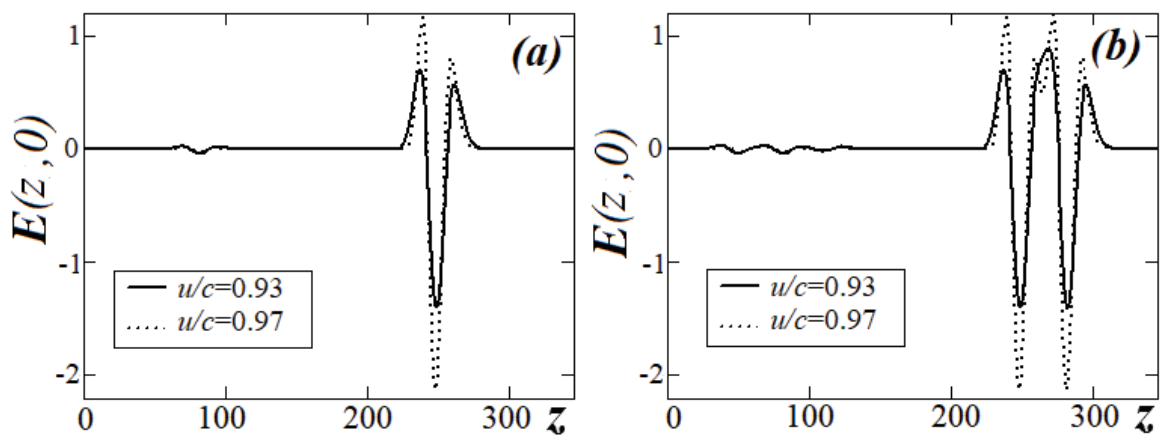

Fig. 2. The electric field at various values of pulse velocity: a) $n=1$; b) $n=2$.

A three-dimensional few-cycle optical pulse propagates with a damped amplitude and with the shape conservation, which is due to the balance of losses of its energy and the energy scattering caused by dissipation. The velocity of the pulse at the entrance to the sample has a strong effect on its evolution.

This work was supported by the Ministry of Education and Sciences of the Russian Federation (project no. MK-4562.2016.2, project no. 2.852.2017/4.6).

\section{References}

[1] S. Lebeque, O. Eriksson, Phys. Rev. B 79, 115409 (2009)

[2] A.V. Zhukov, R. Bouffanais, N.N. Konobeeva, M.B. Belonenko, Phys. Lett. A 380, 3117 (2016)

[3] M. Ezawa, J. Phys. Soc. Jpn. 84, 121003 (2015)

[4] A.V. Zhukov, R. Bouffanais, E.G. Fedorov, M.B. Belonenko, J. Appl. Phys. 114, 143106 (2013) 